\title{
Leibniz und der Eklektizismus
}

Ulrich Johannes Schneider

\section{Text und Kontext}

Die Erforschung der deutschen Philosophie um 1700 hat in den vergangenen beiden Jahrzehnten starke Impulse erfahren; es wurden bisher unbekannte Diskussionen und Richtungen entdeckt, darunter der Eklektizismus. Der Eklektizismus war in den letzten Jahrzehnten des 17. und den ersten Jahrzehnten des 18. Jahrhunderts Gegenstand einer europaweiten Debatte, besonders unter Deutschlands Universitätsphilosophen. Womit sich die jüngst aufgeblühte Eklektizismus-Forschung beschäftigt, sind nicht Lehrbücher oder Methodentraktate, sondern so etwas wie eine philosophia in dissertatio, eine in vielen kleineren Schriften lebhaft geführte Auseinandersetzung über Definition und Anwendungsbereiche des Eklektizismus. Inhaltlich geht es um eine Neubestimmung der Philosophie im versöhnenden Ausgleich der Ansprüche von Antike und Moderne, von Aristotelismus und Cartesianismus, deren Konflikt die Problemlage der Akademiker um 1700 allgemein charakterisierte. ${ }^{1}$ Eine bekannte Figur unter denen, die sich positiv auf den Eklektizismus beziehen, ist Christian Thomasius, dessen Denken in den letzten Jahren ebenfalls verstärkt studiert wurde. ${ }^{2}$ Viele weniger bekannte Figuren der Eklektizismus-Debatte sind durch kontextualisierende Forscher wie Horst Dreitzel oder Michael Albrecht ans Tageslicht geholt worden, in deren Arbeiten das doxographische Bild der Philosophie im späten 17. und im frühen 18. Jahrhundert geweitet und gedehnt wird, so daß heute eine breite (nicht nur philosophische) Kultur das historiographische Feld dort belebt, wo traditionell Wilhelm Gottfried Leibniz und Christian Wolff die Geschichte der Philoso-

1 H. Holzhey, Philosophie als Eklektik, in: Studia Leibnitiana 15 (1983), S. 19-29.

2 Vgl. Christian Thomasius (1655 - 1728). Interpretationen zu Werk und Wirkung, hrsg. v. W. Schneiders, Hamburg 1989, und W. Schneiders, Recht, Moral und Liebe. Untersuchungen zur Entwicklung der Moralphilosophie und Naturrechtslehre des 17. Jahrhunderts bei Christian Thomasius, Münster 1960; ders., Naturrecht und Liebesethik. Zur Geschichte der praktischen Philosophie im Hinblick auf Christian Thomasius, Hildesheim 1971; ders., Aufklärung und Philosophie im Hinblick auf Christian Thomasius, Hildesheim 1971; ders., Aufklärung und
Vorurteilskritik, Stuttgart 1983; vgl. ebenfalls ders., Vernünftiger Zweifel und wabre EklekVorurteilskritik, Stuttgart 1983; vgl. ebenfalls ders., Vernünftiger Zweifel und wabre Eklek-
tik. Zur Entstehung des modernen Kritikbegriffs, in: Studia Leibnitiana 17 (1985), S. 142-161. 
phie fast allein repräsentierten. ${ }^{3}$ Ersetzt oder ergänzt der Eklektizismus unser traditionelles Bild der frühmodernen Philosophie?

Christian Wolff ist bekannt für seine Systematisierung philosophischer Argumentationen und Disziplinen, er gilt als der eigentliche Vorläufer Kants und damit des modernen wissenschaftlichen Denkens, das sich selbst als methodisch streng und sachlich differenziert behauptet. Die mit Wolff einsetzende Bemühung um enzyklopädische Kompetenz führte die Philosophie des späten 18. Jahrhunderts zum Kontakt mit anderen Wissenschaften, und eben in dieser Beziehung liegt das Moderne der rationalistischen Philosophie, die im 19. Jahrhundert das gesamte Universitätswesen ideologisch und praktisch mitbestimmen sollte. Von Christian Wolff nun ist das Bekenntnis überliefert, er sei als Systematiker der beste Eklektiker. ${ }^{4}$ So stellt man sich die Geschichte insgesamt vor: Der Eklektizismus war in dem Moment philosophisch abgetan, als ein Wille zum sachhaltigen Begriff der Philosophie sich durchsetzte, der die Frage nach dem Vorbild - Aristoteles oder Descartes - obsolet machte. Was man im späten 18. Jahrhundert an Bekenntnissen zum Eklektizismus findet oder was man in den Philosophiegeschichten als zeitgenössische Eklektik bezeichnet findet, ist philosophiehistorisch marginalisiert. ${ }^{5}$ Der Wolffianismus und erst recht der Kantianismus sind Formen wissenschaftlicher Philosophie, die kein „Urteil" mehr an eine „Auswahl" bindet und kein „Prüfen" mehr an ein "Aufbewahren".

Was also durch die neuere Forschung zum Eklektizismus sichtbar wird, scheint eine Art hintergründige Ergänzung unseres philosophiehistorischen Wissens zu sein, Dokumentation einer zeitgenössisch verbreiteten Denkhaltung, die sich nicht durchgesetzt hat und die für spätere Generationen - einschließlich der unsrigen - eher historisch und weniger philosophisch interessant ist. Anders gesagt: Der Eklektizismus ist derjenige Kontext, aus dem sich die moderne Philosophie emanzipiert. Die Absage von Wolff an den Eklektizismus kann man zwar als Hinweis auf eine gewisse Aktualität dieser Richtung werten, aber es bleibt eine Absage: Der Text der eigentlichen Philosophie wird anders geschrieben. Dazu paßt die durch die jüngere Forschung gut belegte Tatsache, daß „kein eklektisches Werk" im eigentlichen Sinne unter den zahlreichen Schriften zum Thema zu finden ist und daß viele der expliziten Befürworter des Eklektizismus - wie Johann Franz Budde, Johann Christoph Sturm oder auch Thomasius selbst - in späteren Jahren ihrer Karriere

3 H. Dreitzel, Zur Entwicklung und Eigenart der, eklektischen' Philosophie, in: Zeitschrift für historische Forschung 18 (1991), S. 281-343; M. Albrecht, Eklektik. Eine Begriffsgeschichte mit Hinweis auf die Philosophie- und Wissenschaftsgeschichte, Stuttgart 1995

4 Chr. Wolff, Vernünfftige Gedanken von Gott..., Teil 2, Frankfurt am Main 1724, 377 (Werke, 1. Reihe, Bd. 3, 1983), S. 411; vgl. dazu Albrecht, Eklektik, a.a.O., S. 531.

5 Vgl. W. Schmidt-Biggemann, Theodizee und Tatsachen. Das philosophische Profil der deutschen Aufklärung, Frankfurt a. M. 1988, bes. S. 203-222 (Über die Reichweite des Eklektizismus). darauf nicht mehr zurückkamen: als ob es eine Sache der Jugend gewesen sei, ein Konzept des Protests.

Die Trennung von Text (die moderne rationalistische Philosophie um 1700) und Kontext (der Eklektizismus zur selben Zeit) scheint mit Rücksicht auf Leibniz nicht gleichermaßen durchführbar. Die Versuche, ihn als Eklektiker zu bezeichnen, datieren aus einer Zeit lange vor der Diskussion um die jüngst näher erforschten Eklektiker. Eine ältere philosophiehistorische Forschung, einsetzend im späten 19. Jahrhundert, als die rationalistischen Modelle enzyklopädischen Denkens an den Universitäten ihre Tragfähigkeit verloren, hat gelegentlich zur These gefunden, Leibniz habe "eklektisch" oder "konziliatorisch“, ja selbst „synkreristisch“ gedacht. Angesichts der jüngsten Forschung zum Eklektizismus und dessen Attraktivität zu eben der Zeit, in der Leibniz lebte, könnte man nun versucht sein herauszufinden, welche Bedeutung er für Leibniz gehabt haben mag. Kann die neuere Eklektizismusforschung das Bild von Leibniz berühren, gibt es eine Überlagerung von Text und Kontext? Die Leibniz-Forschung scheint diese Frage noch nicht gestellt zu haben, wofür es gute Gründe gibt. Zum einen ist die Edition des philosophischen Werkes jüngst erst bis in die achtziger Jahre des 17. Jahrhunderts vorgedrungen, so daß die Kontaktnahmen des großen Denkers mit seinen philosophischen Zeitgenossen noch nicht in hinreichender Dichte rekapituliert werden können. Zum anderen ist Leibniz als systematischer Denker von sämtlichen neuerdings als Eklektiker thematisierten Denkern unterschieden. Es scheint, daß sich der Text der Leibniz'schen Spekulation über Gott und Welt nur schlecht mit dem Kontext eines weitgehend universitären Eklektizismus verbinden läßt.

Was sind eigentlich die philosophiehistorischen Optionen für die Beschreibung von Text und Kontext bei Leibniz? Hans Poser hat einmal die Unterscheidung von rationaler und historischer Rekonstruktion philosophischen Denkens am Beispiel von Leibniz und seinem Entwurf einer universalen Logik problematisiert; dabei ging es um die Möglichkeiten einer rationalen Rekonstruktion. ${ }^{6}$ Im Unterschied dazu geht es im Verhältnis von Leibniz zum Eklektizismus eher um die Bandbreite der historischen Rekonstruktion. Die Fortschritte der neueren Philosophiegeschichtserkundungen sind eher literaturhistorisch extensiv als philosophiehistorisch exklusiv, womit der rationale Ertrag der Philosophiegeschichtsschreibung neu bestimmt werden muß. Wo einmal "das Historische" der Philosophie unmittelbar systematische Fragen aufzuwerfen schien', stellen sich in historischer Annäherung eher Strategieprobleme des philosophischen Schreibens, wie sie im Gefolge der Arbeiten

6 H. Poser, Philosophiegeschichte und rationale Rekonstruktion. Wert und Grenze einer Methode, in: Studia Leibnitiana 3 (1971), S. 67-76.

7 Vgl. U. J. Schneider, Ein, Dilemma tiefster Schwierigkeiten: Zur Theorie der Philosophiegeschichte im 20. Jahrbundert, in: Philosophiegeschichte und Hermeneutik, hrsg. v. K.-D. Eichler/V. Caysa (Leipziger Schriften zur Philosophie Bd. 5), Leipzig 1996, S. 46-69. 
von Leo Strauss (noch zu wenig) durchgeführt werden ${ }^{8}$, oder auch Positionsbestimmungen versteckter intellektueller Haltungen, wie in den von Richard Popkin inaugurierten Studien. ${ }^{9}$ Die folgenden Überlegungen gehen nicht so weit, die Möglichkeiten eines historischen Verstehens von Leibniz allgemein $\mathrm{zu}$ untersuchen, sondern versuchen nur das neuerdings erreichte Verständnis des Eklektizismus daraufhin zu befragen, was es für ein Verständnis von Leibniz bedeutet. Das Wissen um den Kontext sollte doch das Wissen um den Text berühren, wenn denn die philosophiehistorische Arbeit (und um mehr geht es hier nicht) in beiden Forschungsrichtungen eine sachliche Gemeinsamkeit wahrt: die historisch angemessene Beschreibung von Philosophie.

Ob Leibniz Eklektiker war oder nicht, ist damit nicht allein eine Frage an seine Schriften und Briefe, in denen er sich dazu explizit äußerte, es ist auch eine methodische Frage der philosophiehistorischen Arbeit. Läßt sich Leibniz überhaupt mit denselben Methoden begreifen wie seine weniger bekannten oder gar völlig vergessenen Zeitgenossen? Allgemein gewendet kann die Frage auch lauten: Worin besteht der Gegensatz zwischen der ideen- und begriffsgeschichtlichen Konzeption der Philosophiegeschichte, wie sie das noch unabgeschlossene, aber bereits gelegentlich veraltete „Historische Wörterbuch der Philosophie" dokumentiert, und der "synthetisierenden" Konzeption des neuesten „Überweg“, der ganz in die Breite zieht ${ }^{10}$, was vordem durch doxographische Sequenzierung (Reihung von Positionen) begriffen wurde, und wofür etwa die simple Dichotomie von Empirismus und Rationalismus ein beliebtes Modell abgab? ${ }^{11}$ Leibniz ist in vielerlei Hinsicht das Kreuz der Philosophiegeschichte, oder die Rose inmitten desselben, um ein Hegelsches Bild zu bemühen: an seiner Philosophie, die ohne eigentliches Buch geblieben ist, hat unser Verstehen große Schwierigkeiten, insbesondere der Feststellung einer Beziehung zwischen geistiger Aktivität und "Werk“, zwischen überlieferten Spuren und "Sinn“, zwischen veranlaßter Denkbewegung und "reiner" Überlegung. Diese Schwierigkeiten sind sicher bei anderen Figuren des modernen Philosophierens nicht geringer, aber historisch gesehen gibt es hier eine gewisse Nähe zu den von der Eklektizismusforschung thematisierten

8 Vgl. H. Meier, Die Denkbewegung von Leo Strauss. Die Geschichte der Philosophie und die Intention des Philosophen, Stuttgart 1996.

9 Vgl. die beiden Festschriften für Richard Popkin (mit Literaturangaben): The sceptical mode in modern philosophy. Essays in honor of Richard H. Popkin, hrsg. v. R. A Watson, Dordrecht 1988; "Everything connects": in conference with Richard H. Popkin. Essays in his bonour, hrsg. v. J. E. Force, Leiden 1999.

10 Die Philosophie des 17. Jahrhunderts: Deutschland [darin über Leibniz S. 997-1159], hrsg. v. H. Holzhey, Basel 2001 (Grundriß der Geschichte der Philosophie, begründet von F. Überweg, völlig neubearbeitete Ausgabe, Band 4).

11 Uber die durchaus uneindeutige Position von Leibniz zwischen Empirismus und Rationalismus vgl. H.-J. Engfer, Empirismus versus Rationalismus? Kritik eines philosophiebistorischen Schemas, Paderborn 1996, S. 158-183.
Denkern, die sämtlich das Philosophieren nicht ins "Werk", sondern in die Arbeit des Nachdenkens setzten.

\section{Eklektisches Denken}

Die neueren Arbeiten zum Eklektizismus in der Philosophie fördern nicht eigentlich neues Textmaterial zutage; sie bewirken eher eine Umbewertung der philosophischen "Gipfelgeschichte". Eklektisches Philosophieren im 17. und 18. Jahrhundert mußte, nicht anders als die Popularaufklärung in Deutschland oder die Philosophie von Victor Cousin im frühen 19. Jahrhundert, "gerettet" werden, indem man die etablierten Vorurteile gegen den Eklektizismus durch ein zeitgemäßes Verstehen aufzuheben versuchte. Historische Entschuldigungsversuche sind inzwischen zu einer selbständigen Form der philosophiehistorischen Aufmerksamkeit gereift, und unser Wissen über das, was Eklektizismus vor und nach 1700 genannt wurde und was sich als solcher behauptete, ist enorm gewachsen. Man vergegenwärtige sich nur die Diskrepanz zwischen dem unzulänglichen Kurzartikel zum Eklektizismus aus dem Historischen Wörterbuch der Philosophie von 1972 und der 1995 erschienenen Habilitationsschrift von Michael Albrecht, die ein erschöpfendes Inventar der einschlägigen Literatur bietet und selbst in diesem Rahmen vieles nur anreißen kann. ${ }^{12}$ Auch ist das Ende des ganzen Eklektizismus-Themas noch unerklärt: War das, was in der ersten Hälfte des 18. Jahrhunderts rasch zum Erliegen kommt, tatsächlich nur eine "Schwundstufe" topischen Denkens, wie Wilhelm Schmidt-Biggemann den Eklektizismus nannte? ${ }^{13}$ Statt gedankliche Erschöpfung zu vermuten, könnte man das eklektische Denken auch als einen Ansatz zur Historisierung der Philosophie verstehen: so hat es etwa in der Philosophiegeschichtsschreibung eine Geltung erlangt, die nicht "systematisch" erledigt werden kann. ${ }^{14}$

Es gibt im 17. Jahrhundert mehrere Spielarten des Eklektizismus, die explizit vertreten werden (und nur diese sind Gegenstand der jüngsten Entdekkungen, denn implizite Eklektik ließ und läßt sich je nach Interpretation immer zusprechen ${ }^{15}$ ). Bei Johann Christoph Sturm etwa handelt es sich um eine

12 Art. "Eklektizismus", in: Historisches Wörterbuch der Philosophie, Band 2: D-F, Basel 1972, Sp. 431 f.; Albrecht, Eklektik, a.a.O

13 W. Schmidt-Biggemann, Topica Universalis. Eine Modellgeschichte humanistischer und barocker Wissenschaft, Hamburg 1983, S. 249-292.

$14 \mathrm{Vgl}$. U. J. Schneider, Eclecticism and the History of Philosophy, in: History and the Disciplines. The Reclassification of Knowledge in Early Modern Europe, hrsg. v. D. Kelley, University of Rochester Press 1997, S. 83-101

15 Albrecht hat mit dem selbstgesteckten Ziel, nur explizite Eklektiker aufzunehmen, auch seine Schwierigkeiten. Vgl. U. J. Schneider/M. Albrecht, Eklektik, Stuttgart 1994, in: Philosophische Rundschau 43 (1996), 180-183; U. J. Schneider, Eclecticism reconsidered, in: Journal of the History of Ideas 59.1 (1998), S. 173-182. 
Variante der philosophia novantiqua und dient der Rechtfertigung experimenteller Physik, bei Christian Thomasius geht es um intellektuelle Selbständigkeit, um das iudicium und um Einsicht in die Endlichkeit menschlicher Einsicht. Bei dem Philosophiehistoriker Johann Jakob Brucker wiederum ist der Eklektizismus nicht nur die modernste Form der Philosophie, sondern auch die einzig praktikable Haltung des Philosophiehistorikers, der sich keiner Parteilichkeit zeihen lassen möchte. ${ }^{16}$ Eklektizismus ist eine Forschungsmethodologie, hat eine Ethik und erlaubt geisteshistorisches Verstehen - das sind Grundzüge einer Charakteristik, die sich in vielen Varianten durch viele Schriften der Leibniz-Zeit belegen läßt. ${ }^{17}$

Der Eklektizismus wird häufig so dargestellt, als ersetze er die Entgegensetzung eines "Entweder-Odex" durch die Gleichgültigkeit eines „SowohlAls Auch". Und tatsächlich bildet der Begriff der Versöhnung (conciliatio) einen der Schlüsselbegriffe des Eklektizismus. Mit einer Ausnahme identifizieren alle deutschen Eklektiker der Zeit um 1700 den Titel des Conciliator mit dem des Eklektikers, denn für sie ist die Annahme der tiefen Einheit des Wissens und der Wahrheit Ziel und Voraussetzung der hermeneutischen Arbeit. ${ }^{18}$ Gegen den Kriegszustand unter den Philosophen ruft Sturm zum Frieden und zum Langmut auf; er warnt vor vorschneller Zurückweisung. Thomasius betont, die eklektische Philosophie sei nicht parteiisch, sondern mit gleicher Liebe allen zugetan. Gotffried Olearius schließt daran an, wenn er fordert, alle müssten gehört werden (omnes audiantur). ${ }^{19}$ So erstreckt sich die Verpflichtung auf Wahrheit auch auf die Bibliothek alter Weisheit, - wie umgekehrt darin der wissenschaftliche Rigorismus der Neuzeit den antiken Titel studio sapientiae erhält. ${ }^{20}$

Die Vermittlung der conciliatio ist aber kein versöhnlerisches Geschäft, das Unterschiede zu ignorieren trachtet - im Gegenteil. Vermittlung setzt Anerkennung der Verschiedenheit voraus. Sie strebt nur danach, die Verschie-

16 Vgl. U. J. Schneider, Das Eklektizismus-Problem der Philosophiegeschichte, in: Johann Jakob Brucker (1696-1770). Philosoph und Historiker der europäischen Aufklärung, hrsg. v. Th. Stammen/W. Schmidt-Biggemann, Berlin 1998, S. 135-158.

17 Die folgenden fünf Absätze folgen den Schlußpassagen in: U. J. Schneider, Ober den philosophischen Eklektizismus, in: Nach der Postmoderne, hrsg. v. A. Steffens, Düsseldorf 1992, S. 219-223.

18 Vgl. J. F. Budde, Elementa Philosophiae Instrumentalis sen Institutionem Philosophiae Eclecticae, Halle (1703), 8. Aufl. 1722, Kap.VI, §XLIV, S. 96 f

19 J. Chr. Sturm, Philosophia Eclectica, Altdorf 1686, Kap. II, S. 26 ff.; Chr. Thomasius, Einleitung in die Hofphilosophie, Frankfurt, Leipzig 1719, \$92; G. Olearius, De philosophia eclectica, in: Historia philosophiae vitas opiniones resque gestas et dicta philosophorum sectae cuiusuis complexa autore Thoma Stanleio, Leipzig 1711 [2. Auflage Venedig 1731], S. 1218 (Ausgabe Venedig 1731: Bd. III, S. 357).

20 Vgl. A. Wesenfeld, Dissertationes Philosophicae Quatuor Materiae Selectioris de Philosophia Sectaria et Electiva, Frankfurt 1694, Diss. IV, \$I (S. 3); G. Olearius, De philosophia eclectica, a.a.O., S. 1207 (Ausgabe Venedig 1731: Bd. III, S. 345). denheit mittels eines „Dritten“, der Wahrheit, zu begreifen. ${ }^{21}$ In Bezug auf die Wahrheit sind weder Überlieferung noch Erfahrung mehr als Annäherungen. So gibt es keinen Kanon des Eklektizismus, obwohl man einen „eklektischen Kursus" konzipieren kann, wie ihn etwa der Logikprofessor Feuerlin in seinem Wissenschaftssystem entwirft..22 Wesenfeld schreibt, im Grunde kenne der Eklektiker kein Corpus bestimmter Wahrheiten, sondern nur die fortgesetzte Prüfung. ${ }^{23}$ So ist für den Eklektiker die Wahrheit nicht gestreut, noch muß sie zusammengesetzt werden. Sie ist weder heidnisch noch christlich noch sonstwie festgelegt. In diesem Sinn ist zu verstehen, daß der Eklektizismus kein Programm der Konstruktion, sondern eines der Rekonstruktion formuliert. Die Tugend des Eklektikers besteht darin, die Wahrheit zu enthüllen oder sie in ihrer Unverhülltheit offenbar zu machen.

In letzter Instanz dient die dem Eklektizismus inhärente Hermeneutik dazu, das Leben beruhigter und das Denken harmonischer zu gestalten. ${ }^{24}$ Darum wird der Eklektizismus auch niemals ganz dem Vorwurf entgehen können, dasjenige zusammenbringen zu wollen, was nicht zusammengebracht werden kann. Bereits in der Debatte des 17. Jahrhunderts versuchen sich die Partisanen des Eklektizismus von den "Synkretisten" abzusetzen, die das griechische Denken mit dem christlichen, oder die Philosophie Platons mit der des Aristoteles verbinden wollten. Gleichwohl bleibt die Option des Synkretismus gegenwärtig, denn dieser ist nichts anderes als ein übertriebener Eklektizismus, bei dem das Resultat der Interpretation dogmatisiert wird. Sobald die Rekonstruktion der Wahrheit als etwas über der rekonstruierten Wahrheit Stehendes behauptet wird, fällt auch der Eklektizismus ins Sektierertum.

Diese Gefahr für die hermeneutisch orientierte methodische Philosophie droht allerdings nicht allein dem Eklektizismus. Nur ist dieser hier besonders verwundbar, weil er definitionsgemäß keinen eigenen "Inhalt" besitzt, $d . h$. keine unabhängig von der Methode der Interpretation formulierbaren Lehrsätze. Die deutschen Eklektiker versuchen dieser Gefahr mit einem eigenwilligen und überraschenden Bekenntnis zu begegnen: Sie leugnen, daß der Eklektizismus gelehrt werden könne. Thomasius unterscheidet die Philosophie "der Schule" strikt von der Philosophie "des Hofs" (d.h. der Welt) und ordnet den Eklekrizismus nur letzterer zu. ${ }^{25}$ Der Helmstedter Anwalt des Eklektizismus Christoph August Heumann folgt ihm darin und empfiehlt für die

21 Vgl. J. B. Röschel, Exercitatio Academica ex Historia Philosophica De Philosophia Conciliatrice, Wittenberg 1692, $₫$ XVI.

22 J. W. Feuerlin, Cursus philosophiae eclecticae, Altdorf 1727.

23 Vgl. A. Wesenfeld, Dissertationes Philosophicae Quatuor, a.a.O., Diss. III, Kap. I, \VI.

24 Vgl. J. F. Schneider, Dissertatio academica de Philosophia Eclectica Civilem Vitae Conversationem non Impediente, sed Adiuvante, Halle 1717

$25 \mathrm{Vgl}$. Chr. Thomasius, Hofphilosophie, a.a.O., \$ 95 
Schule die aristotelisch-scholastische Philosophie, Budde desgleichen. ${ }^{26}$ So zeigen sich die Verteidiger des freien Urteils zurückhaltend im Blick auf die mögliche Verwendung des Eklektizismus. Weder kann es davon einen unmittelbaren noch einen universellen Gebrauch geben. Der Eklektizismus bleibt zuletzt eine unvermittelbare philosophische Haltung.

Diese Unbestimmtheit des philosophischen Eklektizismus ist von Anfang an als seine Schwäche angesehen worden; man könnte sie aber auch als eine Folge seines programmatischen Charakters verstehen. Während er einerseits Lebenspraxis und spekulatives Denken zu vermitteln sucht, kann der Eklektiker andererseits keine Pädagogik begründen, ohne in den Widerspruch zur eigenen methodischen Voraussetzung zu geraten. Denn die Kritik dogmatisierter Denkformen darf auch vor der Arbeit des Eklektikers nicht verstummen. Es ist im Sinne solcher radikalen Subversion, wenn Wesenfeld betont, daß es kein Ende des Denkens (terminum cognitionis) geben könne, wohl aber dessen künftiger Fortschritt angenommen werden könne. ${ }^{27}$ Und Thomasius schreibt nicht weniger radikal, daß die Philosophie niemals eine, niemals eine einheitliche sein könne:

„Eine einzige Philosophie, die in dem einzigen Stande bleiben kann, ist nicht so beschaffen, daß sie des Nahmens einer wahren Philosophie werth sey, derohalben sollen wir mit einer andern zufrieden seyn." 28

Eklektizismus hieß also in dieser Zeit einer "dogmatischen Krise" (um eine Formulierung von Paul Hazard umzumünzen) die im Grunde unmögliche Position einer Positionsfreiheit; es war der Titel eines Versprechens, einer Hoffnung auf Kritik - weit eher als die Einlösung dieses Versprechens oder die Durchführung von Kritik.

\section{Eklektizismus bei Leibniz}

In der gegenwärtigen Darstellung der Philosophie von Leibniz spielt der Eklektizismus keine Rolle, man muß dafür auf ältere Interpretationen zurückgreifen. So war 1860 für Jean Félix Nourrisson der „Eklektizismus von Leibniz" eine Folge der Tatsache, daß Leibniz die Geschichte der Philosophie nicht wie Descartes gänzlich verwarf, weil Leibniz durch seine Erziehung kenntnisreicher war, durch sein "génie" aufgeschlossener gegenüber fremden Ansätzen und weil sein Ehrgeiz der Versöhnung galt, in theoretischen wie in

26 Chr. A. Heumann, Programma de Optima Secta Philosophorum [1720], in: Poecile sive Epistolae miscellaneae, Teil I, Buch I, Halle 1722, S. 133ff; vgl. J. F. Budde, Philosophischer Diskurs von dem Unterschied der Welt- und Schul-Gelabrtheit [1709] (= Einleitung zu): Martin Musig, Licht der Wahrheit, in denen nöthigsten Stücken der wahren Gelehrsamkeit, zur Erkänntnis menschlicher und göttlicher Dinge, 1.Teil, 2. Aufl., Frankfurt und Leipzig 1716

27 A. Wesenfeld, Dissertationes Philosophicae Quatuor, a.a.O., Diss. IV, \$XIV (S. 24).

28 Vgl. Chr. Thomasius, Hofphilosophie, a.a.O., \$98. praktischen Belangen. ${ }^{29}$ Nourrisson unterscheidet zwei Eklektizismen bei Leibniz: einen der Versöhnung bereits vorliegender Ansätze („,conciliation“) und einen, der sich auf vorliegende Ansätze lediglich stützt, um dadurch weiterzukommen (nach der Devise „plus ultra").30 Leibniz prüft nicht nur, er benutzt, was er für brauchbar hält. Das sei klarerweise nicht mit dem Synkretismus zu verwechseln, den Nourrisson im engen (katholischen) Sinn als christliche Ausrichtung der Philosophie definiert, und bedeute auch nicht, daß Leibniz keine Kritik übe. Leibniz ist großzügig, tolerant, aber nicht dogmatisch: Er ist gegen das Sektierertum. ${ }^{31}$

a) Die Wahrheit in fremder Formulierung. Wenn man zu dieser Interpretation Belege sammeln will, muß man Leibniz als Kritiker von dogmatischer Einseitigkeit und als Einlöser früherer Philosophien thematisieren. Was dann Eklektizismus heißen kann, schließt die Insistenz auf Kenntnisnahme ein. Schon Nourisson geht auf Leibnizens Kritik an Descartes und dessen Verachtung der alten Philosophen ausführlich ein und kann zeigen, daß Leibniz sich nicht gegen die Notwendigkeit einer (auch kritischen oder sogar negativen) Beurteilung anderer Philosophie ausspricht, sondern vor der antizipierten Sicherheit des eigenen Urteils warnt. Leibniz liest anders als Descartes, gewinnbringender, er liest mit Hoffnung auf Entdeckung und ohne Furcht vor Verwirrung. Das vielzitierte "Gold“, das Leibniz in aller vergangenen Philosophie, selbst in der Scholastik, finden zu können mutmaßte ${ }^{32}$, ist der Lohn seiner zurückgenommenen Haltung, die einlassend und nicht herablassend ist. Und hier ist eine Nähe zum Eklektizismus seiner Zeit durchaus vorhanden, denn auch dieser legte großen Wert auf Einlassung und Interpretation. Der Frankfurter Metaphysikprofessor Arnold Wesenfeld etwa schreibt 1694: "Die Eklektiker halten so wenig dafür, frei zu philosophieren bedeute nichts anderes als den Sekten abzuschwören, daß sie die Freiheit des Denkens vielmehr mit dem Respekt für die Schriften und Lehren der anderen verbinden." ${ }^{33}$ In den Neuen Abhandlungen hat Leibniz seine Haltung so ausgedrückt:

29 J. F. Nourrisson, La philosophie de Leibniz, Paris 1860, S. 81-101 (De l'éclectisme de Leibniz), bes. S. $89 \mathrm{ff}$.

30 Ebd., S. $93,94$.

31 Ebd., S. $95,97$.

G. W. Leibniz, Neue Abhandlungen über den menschlichen Verstand, übers. v. Ernst Cassirer [1915], Hamburg 1996, S. 462, vgl. ders., Metaphysische Abhandlung, in: Hauptschriften zur Grundlegung der Philosophie, hrsg. v. Ernst Cassirer [künftig zitiert als HS] Hamburg 1996, S. 353 f.; ders., Auflälarung der Schwierigkeiten, die Herr Bayle in dem "Neuen System der Vereinigung von Seele und Körper" gefunden hat, in: HS, S. 470, sowie den Brief von Leibniz an Remond vom 26.8.1714, in: HS, S. $633 \mathrm{f}$, 33 A. Wesenfeld, Dissertationes Philosophicae Quatuor, a.a.O., Diss. IV, $\$$ XVII (S. 30): Nec Ec-
lecticorum assertio est, "liberè philosophari (absolutè) nihil aliud esse quàm nulli se sectae etc. tradere"; sed tantùm de libertate sentiendi in respectu ad aliorum scripta et dogmata 
"Nicht als ob nicht sehr oft wirklich Grund dafür bestände, die Meinungen anderer zu kritisieren, aber man muß dies im Geiste der Billigkeit und des Mitleids für die menschliche Schwäche tun. " ${ }^{34}$

Leibniz hat bekanntlich an vielen Stellen bezeugt, daß er "Billigkeit" üben kann und Autoren gegen Fehlinterpretationen in Schutz nimmt. ${ }^{35}$ Auch wenn Leibniz gelegentlich auf die "nackte Wahrheit" hoff ${ }^{36}$, möchte man meinen, er habe eine grundsätzlich hermeneutische Haltung, die allein am Ausdruck, an der Erscheinung, an der Repräsentation dasjenige abliest, was als Wahrheit gelten kann, weil diese als solche aber direkt nicht zugänglich ist. Eine Bemerkung aus den Eingangspassagen der Neuen Abhandlungen ist bezeichnend, wo sich die Gesprächspartner darauf einigen, die Thesen des Autors (bekanntlich John Locke) in dessen eigener Formulierung und nicht in irgendeiner abstrahierenden Abkürzung zu diskutieren. Dann sagt Theophilus zu Philaletes: „Ich billige Ihren Vorschlag. Hier ist das Buch. ${ }^{\text {377 }}$ Von dieser Verpflichtung auf die gegebene Formulierung her versteht sich die Betonung der Notwendigkeit von "Kritik" und "Interpretation" beim Umgang mit fremden Äußerungen; von daher wird bei Leibniz aber auch die feste Hoffnung plausibel, alles Alte und Andere zu überwinden: „Denn es hängt von uns ab, die Bedeutungen, wenigstens in der Sprache der Wissenschaft, festzulegen und sich darüber zu einigen ...." ${ }^{38}$ Diese hermeneutische Haltung hat nichts prinzipiell Bewahrendes an sich, sondern etwas Transformierendes. ${ }^{39}$ Und sie erlaubt den Fortschritt, wie ein weiteres Zitat aus dem Eingang zu den Neuen Abhandlungen belegen kann:

„Ich bin auf ein neues System gestoßen [...] Seitdem glaube ich, das innere Wesen der Dinge in einem neuen Lichte zu sehen. Dieses System scheint Platon mit Demokrit, Aristoteles mit Descartes, die Scholastiker mit den Neueren, die Theologie und Moral mit der Vernunft zu versöhnen. Von allen Seiten scheint es das Beste zu nehmen, und dann weiter fortzuschreiten, als man jemals gekommen ist." 40

Für Leibniz ist systematisches Fortschreiten besser durch Einbezug bereits formulierten Wissens als durch generellen Ausschluß desselben erreichbar (das wäre Dogmatismus und Sektierertum), und das ist in der Tat auch die geteilte Meinung der modernen Eklektiker, etwa in der Formulierung von

34 G. W. Leibniz, Neue Abhandlungen, a.a.O., S. 500

35 Vgl. ebd., S. 356 (zu Aristoteles), S. 444 (über die Schulphilosophen) und S. 492 (zu Spinoza).

36 Vgl. ders., Versuche in der Theodicée über die Güte Gottes, die Freibeit des Menschen und den Ursprung des Übels [1710], übers. v. A. Buchenau [1925], Hamburg 1996, S. 19.

37 G. W. Leibniz, Neue Abbandlungen, a.a.O., S. 34.

38 Vgl. ebd., S. 346 [Übers. leicht verändert].

39 Vgl. A. Heinekamp, Die Rolle der Philosophiegeschichte in Leibniz' Denken, in: Leibniz als Geschichtsforscher, hrsg. v. A. Heinekamp, Wiesbaden 1982 (Studia Leibnitiana Sonderheft 10), S. 114-141, bes. S. 123 und $135-141$

40 G. W. Leibniz, Neue Abhandlungen, a.a.O., S. 31.
Heumann, der 1715 schreibt, es sei der „Erkenntnis der Wahrheit nichts hinderlicher als eingebildete Orthodoxie". ${ }^{41}$

b) Universale Perspektive. Dietrich Mahnke hat sich 1912 in einem längeren Aufsatz mit einem zuvor unveröffentlichten Leibniz-Text über die Typen des Sektierertums auseinandergesetzt und die Leibniz'sche Argumentation gegen jede "Gelehrteneinseitigkeit" analysiert, ${ }^{42}$ Leibniz schreibt in dem zitierten Fragment (vermutlich aus den 1680er Jahren) gegen die verschiedenen und doch gleichermaßen nutzlosen Einseitigkeiten der Philologen, der Mathematiker und Naturwissenschaftler, der Scholastiker und der Cartesianer. ${ }^{43}$ Hochmütig und pedantisch seien alle vier Gruppen, unduldsam gegen Neues und neu Ausgedrücktes. Mahnke hebt hervor, daß das Fragment und andere Zeugnisse vor allem aus Briefen von Leibniz dafür plädieren, die verschiedenen Wissensgebiete und die dazu benutzten Methoden einander befruchten zu lassen, und auch dafür, die einzelnen Wissenschaften selbst vorurteilslos zu betreiben. Universalismus und methodischer Erfindungsreichtum machen hier den nichtfestgelegten Denker aus, der in der technischen und politischen Praxis seines Hofbeamtenlebens vielfach theoretische Kenntnisse einsetzte, wie er umgekehrt „sein diplomatisches Geschick und sein Organisationstalent in den Dienst der Theorie" stellte. ${ }^{44}$ Mahnke nun ist vom Universalismus fasziniert, und diesen empfiehlt er seiner eigenen Zeit. ${ }^{45} \mathrm{Er}$ nennt Leibniz nicht direkt Eklektiker, sondern der Tendenz nach, wie Michael Albrecht beobachtet hat, einen "harmonischen Synthetiker" oder Konziliator. ${ }^{46}$

Was Mahnke zeigt, ist im Grunde die Notwendigkeit des philosophischen Universalismus, eine weite Perspektive einzunehmen und gemäß der vom Apostel Paulus entlehnten eklektischen Maxime "Prüfet alles" (1. Brief an die Thessaloniker, 5, 21) nichts wirklich unberücksichtigt zu lassen. Die Vernunft entdeckt sich durch Zusammenstellung, Überblick, im Ganzen, nicht aus partikularer Perspektive. Das ist genuine Überzeugung vieler Eklektiker der Leibniz-Zeit, vor allem der historisch gebildeten. Ganz in ihrem Sinne gibt Leibniz 1696 eine Aufzählung dessen, was sich alles an Vernunft aus anderen Philosophen nehmen läßt:

41 Chr. A. Heumann, Acta philosophorum, das ist: Gründliche Nachrichten aus der Historia philosophica, nebst beigefügten Urteilen, von denen dahingehörigen alten und neuen Büchern, Magdeburg 1715, Einleitung, Kap. 1, S. 32.

42 D. Mahnke, Leibniz als Gegner der Gelehrteneinseitigkeit, Stade 1912 (Wissenschaftliche Beilage zum Jahresbericht des Königlichen Gymnasiums zu Stade, Ostern 1912).

43 Den Text „Spongia exprobationum seu quod nullum doctrinae verae genus sit contemnendum" gibt Mahnke im Original auf den Seiten 94-100 im Anhang und in freier Übersetzung auf den Seiten 26-33.

44 Ebd., S. 64.

45 Ebd., S. 14 f., S. $91 \mathrm{f}$

46 Vgl. D. Mahnke, Leibnizens Synthese von Universalmathematik und Individualmetaphysik, Halle 1925, Nachdruck Stuttgart 1964, S. 12 f.; M. Albrecht, Eklektik, a.a.O., S. 295. 
„Die geringe substantielle Realität der Sinnen-Dinge, die die Skeptiker, die Zurückführung aller Dinge auf Harmonien oder Zahlen, auf Ideen und Perzeptionen, die die Pythagoreer und Platoniker gelehrt haben, das identische, allumfassende Eine des Parmenides und Plotin, das dennoch allem Spinozismus fern bleibt, die stoische Notwendigkeit, die dennoch mit der Selbsttätigkeit vereinbar ist, die Lebensphilosophie der Kabbalisten und Hermetiker, nach denen es überall Empfindung gibt, die Formen und Entelechien des Aristoteles und der Scholastiker, die trotzdem die mechanische Erklärung aller besonderen Phänomene gemäß Demokrit und den Modernen nicht ausschließen" ...

und endet diese Aufzählung mit den Worten:

$\ldots$ "dies alles findet sich hier wie in einem perspektivischen Zentrum vereinigt, aus dem der Gegenstand - der von jeder anderen Stelle betrachtet wirr erscheint - seine Regelmäßigkeit und die Übereinstimmung seiner Teile erkennen läßt." ${ }^{47}$

Zum Zentrum vorzudringen setzt die genaue Kenntnis der Peripherie voraus, ist im Grunde eine Bewegung der Konzentration als Annäherung an den Kern. Leibniz ist ein großer Kenner von Umständen und Umwegen und hat zugleich immer den Kernbereich des Vernünftigen im Auge, etwa bei seinen Bemühungen um die Wiedervereinigung von katholischer und protestantischer Kirche. So verfaßte er 1698 ein Memorandum für den Kaiserlichen Beauftragten für die Reunionsverhandlungen mit einer Liste von 30 inhaltsleeren Kontroversen zwischen den beiden christlichen Konfessionen und lieferte 1699 im Briefwechsel mit dem französischen Bischof Bossuet 124 historische Argumente gegen die Gültigkeit der Beschlüsse des Konzils von Trient, um (letztlich vergeblich) das Gemeinsame in der Geschichte wiederherzustellen. ${ }^{48}$ Auch die Einigung der protestantischen Kirchen war ihm umwegige Argumente und Hinweise auf den Kernbereich des Gemeinsamen werr ${ }^{49}$, ebenso wie im politischen Bereich das "Gleichgewicht der europäischen Kräfte", was André Robinet zum Gegenstand eines ganzen Buches machen konnte, das den aussöhnenden Leibniz als "politischen Philosophen" und als "philosophischen Politiker" ausweist. ${ }^{50}$ Leibniz ist als Universalist Advokat der Zentralperspektive und aus diesem Grund, nicht aus anthropologischer Einsicht oder religiöser Überzeugung, konziliatorisch eingestellt. Mit dem Eklektizismus seiner Zeit hat das wenig zu tun, weil dort der Universalismus schon in der Philosophie nicht entwickelt ist, geschweige denn in anderen Bereichen. Leibniz könnte als Eklektiker nur in Hinblick auf das Streben nach Aussöhnung bezeichnet werden, ohne Rücksicht auf die Motive oder Strategien dieses Strebens. Aber gibt es ein solches reines Streben?

47 G. W. Leibniz, Aufklärung der Schwierigkeiten, in: HS, S. 470.

48 Vgl. die Dokumente in Foucher de Careil, Oeuvres de Leibniz, 7 Bände, Paris 1859-1875, Nachdruck Hildesheim 1969, Band 2, S. 172-193 und 318-373

49 Vgl. zusammenfassend E. Chr. Hirsch, Der berühmte Herr Leibniz, München 2000, S. $385-$ 388 .

50 A. Robinet, G. W. Leibniz, Le meilleur du monde par la balance de l'Europe, Paris 1994. c) Philosophie als Forschung. In Herman Schmalenbachs Leibniz-Buch von 1921 wird im Schlußkapitel die "prinzipielle Konziliatorik" von Leibniz behandelt, die Schmalenbach auch "Eklektizismus" nennt. ${ }^{51}$ In diesem Buch wird metaphorisch die Charakterisierung von Leibniz als barocker Denker aufgearbeitet, wobei Schmalenbach das Bild des Bauwerks durch dasjenige des Gewebes ersetzen will, das ihm eher erlaubt zu sagen, Leibniz habe die in der Jugend geknüpften Fäden weitergesponnen. „Immerhin liegt bei Leibniz der äußere Tatbestand der Hereinnahme fremder Philosopheme vor", führt Schmalenbach ins Feld, um dann rasch einen "gemeinen" oder "profanen Eklektizismus" bei Leibniz abzuweisen..$^{52}$ Der große Denker habe von frühester Jugend an bewiesen, daß seine Empfänglichkeit für andere Philosopheme und Theorien eine Stärke und keine Schwäche war, und Schmalenbach kann ihn deswegen nur mit Hegel vergleichen. ${ }^{53}$ Dieser höhere Eklektizismus gehe eine sublime Verbindung mit dem "Versöhnen-Wollen" ein, das sowohl der Aufklärungszeit selbst als auch religiösen Anliegen der Zeit unterstellt werden könne. ${ }^{54}$

Die "prinzipielle Konziliatorik" macht allerdings für Schmalenbach aus Leibniz mehr als einen Zeugen der Zeit: Sie „ist ein Produkt aus dem allgemeinen Harmonisierungsbestreben und dem, was wir oben in freilich eingeschränkterer Weise Leibniz' Eklektizismus nannten". ${ }^{55}$ Eklektizismus nährt Harmonismus und Optimismus: So wird angesprochen, was sonst eher beiläufig die "Vielseitigkeit" von Leibniz heißt, wo es wenig Gegensatz und viel Nebeneinander gibt. Auf eine andere Tatsache hatte schon Mahnke hingewiesen: Leibniz hat kaum das hinterlassen, was man ein Werk nennt. Eine Universalität mit tragischem Zug habe Anlaß gegeben, vom "Mangel an Begrenzung" zu sprechen, und berechtigt jedenfalls, eine "Zersplitterung der Kräfte" zu diagnostizieren. ${ }^{56}$ Eklektizismus und Konziliatorik als schwaches Denken avant la lettre, als Beweis unkräftiger Theorie?

Es mag so scheinen, als sei Leibniz ein Entwerfer von immer neuen Ansätzen, ein Konzipierer von unglaublich vielen Notizen und ein Autor von mehreren abgeschlossenen, aber unveröffentlichten Studien, also ein unermüdlicher Schreiber "unsichtbarer Bücher", wie es ihm der unzufriedene Kurfürst von Hannover einmal an den Kopf warf. ${ }^{57}$ Das konziliatorische Element ist in dieser Sichtweise Anzeichen eines gar nicht doktrinfähigen Denkens, das sich als etwas Drittes unter bereits bestehende Kontroversen mischt und durch deren Auflösung eigene Kraft gewinnt, die allerdings unabhängig

51 H. Schmalenbach, Leibniz, München 1921, S. 475 ff.

52 Ebd., S. 476 ff.

53 Ebd., S. 479-482.

54 Ebd., S. 478.

55 Ebd., S. 484; vgl. S. 505

56 D. Mahnke, Leibniz als Gegner der Gelehrteneinseitigkeit, a.a.O., S. $69 \mathrm{ff}$.

57 E. J. Aiton, Gottfried Wilhelm Leibniz. Eine Biographie, Frankfurt a. M. 1991, S. 318 
von dieser Auflösung keine Wirkung zeitigen kann. Es ist in der Tat ein schon früh erhobener Vorwurf gegen den Eklektizismus, er ziele auf inventio nur im topisch-enzyklopädischen Sinn einer Umordnung, nicht im wissenschaftlichen Sinn einer Entdeckung. Leibniz trifft dieser Vorwurf nicht, und er kann ihn nicht treffen, nicht nur, weil Leibniz selbst keinen Eklektizismus propagierte, sondern auch und vor allem, weil die Leibniz'schen Kautelen der gerechten Auslegung, der Unvoreingenommenheit und der Universalperspektive tatsächliche Arbeitsmittel sind, nicht Forderungen oder methodische Mahnungen. Leibniz hat Erkenntnistheorie und Methodenlehre ebenso vernachlässigt wie Reflexionen über Studium und Bildung; er hat die Regeln für das Denken nicht separat formuliert, sondern im Prozeß der Ausarbeitung bestimmter Gedankengänge benannt und in der Anwendung explizit gemacht. Man denke nur an die beiläufige Art, mit der er im philosophischen Spätwerk seinen "Satz des zureichenden Grundes" einführt. ${ }^{58}$ Regeln und Prinzipien sind ihm Arbeitsinstrumente.

So ist auch keiner der Leibniz-Interpreten je so weit gegangen, aus dem fragmentarischen Charakter des Leibniz'schen Werks ein Argument für die Nähe zum Eklektizismus zu machen, was man tun könnte, würde man die entsprechenden Schriften der Zeitgenossen von Leibniz als ebenso viele Zeugnisse dafür nehmen, das Denken mit der Vorüberlegung zu identifizieren, mit dem Essay, der Skizze, dem Konzept. Nach bald dreihundert Jahren Leibniz-Forschung weiß man aber, daß die Beiträge von Leibniz zu den verschiedensten Wissenschaften und Künsten einfach zu wirkmächtig waren, als daß man aus der Zerstreutheit der schriftstellerischen Produktion irgend etwas anderes als methodische Vorsicht und intellektuelle Bescheidenheit und höchstens im einen oder andern Fall auch Ungeschicklichkeit schließen wollte. Es gibt jedenfalls kein philosophisches Prinzip für den zerstreuten Charakter des Werkes von Leibniz, höchstens eine forschungspraktische Maxime, alles durch eigene Einsicht anzueignen und niemals „auf eines Meisters Worte zu schwören", wie ein damals oft benutztes Horaz-Zitat lautet. Und so nimmt es auch nicht wunder, daß Leibniz sich nirgends zum Eklektizismus bekannt hat. Es gibt eine Briefstelle von 1694, wo Leibniz in Bezug auf zeitgenössische Eklektiker wie Wesenfeld differenziert:

„Wir dürfen keine Eklektiker sein wie diejenigen, die sogenannte Gemeinplätze mit ihrem Gegenteil zusammenstellen, oder wie diejenigen, die eine philosophische Geschichte schreiben und eine Lehre exzerpieren, statt sie in Fleisch und Blut zu verwandeln. “59

58 Vgl. G. Deleuze, Die Falte. Leibniz und der Barock, Frankfurt a. M. 1995, S. 75-96; dazu U. J. Schneider, Das Problem der zureichenden Vernunft. Leibniz - Heidegger-Deleuze, in Nihil sine ratione. Mensch, Natur und Technik im Wirken von G. W. Leibniz (Akten des VII. Internationalen Leibniz-Kongresses), hrsg. v. H. Poser, Berlin 2001, S. 1138-1146.

59 G. W. Leibniz, Brief vom Oktober 1694 an Gerhard Meier, in: A I, 10, 592 (Nr. 403); vgl. Albrecht, Eklektik, a. O, S. 295.
Dieses „in Fleisch und Blut verwandeln“ ist in der Eklektizismus-Debatte um 1700 durchaus eine Zielbestimmung, bei Leibniz aber eine Tatsache der beständigen Arbeit. Die Beschäftigung mit dem Eklektizismus, dessen Bedeutung und Tragweite zur Leibniz-Zeit betrifft in der Hauptsache philosophische Programme. Dagegen kann die Forschungsarbeit eines Leibniz, der sich eklektischer Grundsätze bedienen mag, nur um den Preis einer groben Verkürzung eklektisch genannt werden. Anders gesagt, für den forschenden Denker ist Eklektizismus an bestimmten Stellen der Arbeit praktisch unvermeidlich, ohne daß man daraus aber ein Programm ableiten könnte.

Man vergegenwärtige sich noch einmal die Nähe von Leibniz zum „Eklektizismus", wie sie sich aus einem Brief an Remond vom 10. Januar 1714 herauslesen läßt:

"Ich habe von jeher versucht, die Wahrheit, die unter den Ansichten der verschiedenen philosophischen Sekten begraben und verstreut liegt, aufzudecken und mit sich selbst zu vereinigen, und ich glaube, von meiner Seite dazu mitgewirkt zu haben, daß wir hierin einige Schritte vorwärtsgekommen sind."

Das ist das erste eklektizistische Element: die Wahrheit kann in fremder Formulierung entdeckt werden. Im Brief folgt der zitierten Stelle die Schilderung der Lehrjahre, wobei Leibniz die Vielfalt seiner philosophischen Kenntnisse hervorhebt. Am Schluß werden die beiden anderen eklekrizismusähnlichen Elemente benannt: Entwicklung einer überparteilichen Perspektive und Relativierung bzw. Benutzung vorhandener Meinungen im Fortgang der eigenen Studien, d. h. der sachlich motivierten Forschungsarbeit:

"Ich habe gefunden, daß die meisten Sekten in einem guten Teile dessen, was sie positiv behaupten, recht haben, weniger aber in dem, was sie leugnen, was sie schmeichle mir, in die Harmonie der verschiedenen Reiche eingedrungen zu sein und erkannt zu haben, daß beide Parteien recht haben, vorausgesetzt, daß sie gegenseitig ihre Kreise nicht stören, daß also alles in den Naturerscheinungen auf mechanische und metaphysische Weise geschieht, daß aber die Quelle der Mechanik in der Metaphysik liegt. Es war nicht leicht, dieses Geheimnis zu entdecken, weil nur wenige sich die Mühe nehmen, diese beiden Arten von Studien miteinander zu vereinigen." 60

Wenn damit ein Eklektizismus benannt wird, dann einer, der sich auf sich selber anwendet und damit aus der bloßen Empfehlung einer Methode heraustritt: Er wird ethos der philosophischen Praxis. ${ }^{61}$ Zeitgenössisch vergleichbar ist nur die Forderung von Christian Thomasius, durch Unterricht Wider-

60 G. W. Leibniz, HS, S. 623, 624

61 Die eklektischen Begriffe „Überlegung", "Auswahl" und „Entscheidung" sind Begriffe einer Ethik, die Leibniz sozusagen am Rande seiner wissenschaftlichen Projekte entwickels. Vgi M. de Gaudemar, Ethique et morale chez Leibniz, in: Philosophie 39 (1993) S. $60-82$, bes. S. $69 \mathrm{ff}$. 
spruch zu fördern und nicht Gefolgschaft ${ }^{62}$ - mit dem Unterschied, daß es bei Leibniz nicht um Forderungen geht, sondern um Forschung.

\section{Philosophiehistorische Optionen}

In der historischen Rekonstruktion rücken Leibniz und der Eklektizismus der Leibniz-Zeit nur zusammen, wenn man Forschung und Forderung, Programm und Praxis identifiziert. Dann lassen sich Sätze, die Leibniz im Vorübergehen zu anderen, älteren Philosophen macht, im Kontext der Eklektizismus-Diskussion verstehen, wo es grundsätzlich und ausschließlich um das Verhältnis gegenwärtigen Denkens zu anderen (auch hauptsächlich vergangenen) geht. An wenigen Stellen wird dann der Text der Leibniz'schen Philosophie zum Teil dieses Kontextes und läßt sich von daher verstehen. Unter den verschiedenen Interpretationen, die einen „eklektischen" Leibniz entwerfen, gibt es aber keine, die im engeren Sinn eine philosophiehistorische Option darstellt, was abschließend kurz gezeigt werden soll.

Die Rede von philosophiehistorischen Optionen zielt auf die Angemessenheit historischen Verstehens philosophischer Aktivitäten, was sich unabhängig von den Erfordernissen der rationalen Rekonstruktion erörtern läßt. Für kein systematisches Verständnis ist Leibniz sinnvoll als Eklektiker zu bezeichnen, weil - gerade auch nach den jüngsten Untersuchungen des „Eklektizismus-Syndroms" 63 - Eklektizismus gar keine philosophische Position darstellt, sondern so etwas wie eine Art undogmatisches Denken und Problematisieren von Dogmatismus. Die historische Rekonstruktion jedoch muß sich zumindest mit denjenigen Textstellen auseinandersetzen, die dasjenige, was man im historischen Verständnis und auch noch in einem gegenwärtig nicht ungeläufigen Sinn als Eklektizismus bezeichnen kann. Um von dort auf eine Interpretation von Leibniz als Eklektiker zu gelangen, müssen allerdings folgende Zusatzannahmen gelten:

a) der unmittelbare Kontext der Leibniz-Stelle wird ignoriert (etwa die Diskussion einer These) und die Stelle isoliert ausgehoben;

b) die Bezeichnung Eklektizismus oder eklektisch wird vergeben, auch wenn nur einzelne Charakteristika dafür sprechen;

62 Chr. Thomasius, Einleitung in die Vernunft-Lehre, Halle 2. Aufl. 1699 [5. Aufl. 1719], S. 50 [S. 52]: So verlange ich auch nicht von meinen Auditoribus, daß sie meine Sectarii werden sollen, weil ich meine Philosophie für wahr halte, sondern ich will, daß sie mir folgen sollen, wenn sie die Warheit derselben so erkennen werden, als ich; daferne sie aber sehen, und sehr deutlich spüreten, daß ich gefehlet hätte, so inculcire ich ihnen täglich, daß sie alsdenn meine Meynung sollen fahren lassen, gleichwie ich selbst in einen und andern meine Meynung ändere, wenn eine reiffere meditation mir meine Irrthümer zu erkennen giebt.

63 H. Dreitzel, Entwicklung und Eigenart der, eklektischen' Philosophie, S. 330. c) die Abgrenzung von Eklektizismus, Synkretismus und Konziliatorik wird unscharf gemacht, insbesondere im Blick auf verschiedene Wissensgebiete (Philosophie, Naturwissenschaft, Theologie);

Diese Zusatzannahmen finden sich gar nicht selten in der philosophiehistorischen Arbeit gemacht, wo mit Begriffen als übergreifenden Bezeichnungen frei operiert wird. Abgesehen von der Tatsache, daß die historische Verortung von Leibniz als Eklektiker auch ihre Nebenabsichten hatte ${ }^{64}$, läßt sich im Blick auf Leibniz und seine vermutete Nähe zum Eklektizismus nun zusammenfassend sagen, daß hier eine Korrespondenz oder nähere Beziehung nach heutigem Wissensstand aus folgenden Gründen ausscheidet:

a) Die zur Leibniz-Zeit veröffentlichten Schriften zum Eklektizismus sind allesamt problematisierend, was die abstrakte Bestimmung der Philosophie in der Gegenwart anlangt, und hauptsächlich programmatisch, was ihre nähere Zukunft betrifft. Bei Leibniz gibt es weder das abstrakte Problematisieren von Philosophie noch einen vergleichbaren programmatischen Ausblick, sondern so etwas wie einen metaphysisch-forschungspraktischen Optimismus.

b) Die Eklektiker der Leibniz-Zeit haben sich nicht mit Leibniz beschäftigt, weil sie mehrheitlich im dogmatisch-pädagogischen Konfliktfeld der Universitätsphilosophie standen und mit der Konkurrenz von Aristotelismus und Cartesianismus beschäftigt waren. Leibniz selbst hat keinen Kontakt zu dieser Szene, wenn man seine Briefe an Christian Thomasius und seine Bezugnahme auf Arnold Wesenfeld ausnimmt; beide Berührungspunkte zeigen ihn nicht als Sympathisanten des Eklektizismus.

c) Die Felder, auf denen eklektisches, synkretistisches oder konziliatorisches Denken eine Funktion haben kann, sind unter den Eklektikern der Leibniz-Zeit nicht genauer abgegrenzt, so daß in der programmatischen Rede philosophische, wissenschaftliche und theologische Argumente einigermaßen ungeklärt ineinandergreifen. Bei Leibniz sind die verschiedenen Tätigkeitsbereiche in ihrer Arbeitstechnik und Forschungsmethodologie nur schwer aufeinander zu beziehen und in ihrer wechselseitigen Beeinflussung zu gewichten.

Mit den Begriffen "Text" und „Kontext" formuliert: Der eklektische Text wird bei Leibniz weder durch den unmittelbaren Kontext (seine eigenen Schriften) noch durch den mittelbaren (die Schriften seiner Zeitgenossen) bestätigt; die expliziten Bezüge zwischen Text und Kontext sind insgesamt zu

64 So stellt sich Nourrisson in die Linie der explizit eklektischen Philosophie Victor Cousins und benutzt dessen Definition - der Eklektizismus sei nicht Unentschiedenheit zwischen Systemen, sondern entschiedene Wahl eines Systems -, zur Charakterisierung der Einstellung von Leibniz (Nourrisson, S. 96). Und Mahnke gibt offen zu, daß er den universalen Eklektiker Leibniz seiner eigenen Zeit als Vorbild hinstellen möchte; er argumentiert am Vorabend des Ersten Weltkriegs auch nationalistisch: "Gerade die deutschen Gelehrten hätten ihr großes Vorbild Leibniz nie aus dem Auge verlieren dürfen." Vgl. D. Mahnke, Leibniz als Gegner der Gelehrteneinseitigkeit, a.a.O., S. 91; das aktualisierende Schlußwort erstreckt sich über die Seiten 87-93. 
schwach. Man sollte entsprechend die Erforschung beider trennen und könnte damit noch sehr viel weiter fortschreiten, wenn man als philosophiehistorische Optionen auch die Bestimmung rhetorischer und sozialer Kontexte zuließe. Die Probleme des wissenschaftlichen Schriftstellers Leibniz sind mit denen des akademischen Lehrers und öffentlichkeitswirksam tätigen Christian Thomasius nicht vergleichbar: Es fällt in praktischer Hinsicht eine enorme Diskrepanz ins Auge, die allen doxographischen Anschein von Verwandtschaft bedeutend relativiert. Immerhin kann man den älteren und den neueren Bemühungen um den Eklektizismus zugute halten, daß sie an der praktischen Einbettung philosophischer Tätigkeit interessiert waren. Wenn es aber dahin kommt, zu diesem Zweck Textstellen aufeinander zu beziehen, die wie einzelne Thesen aus ihren Schriften herausgehoben werden, dann verliert man eben die Probleme aus dem Blick, die philosophische Tätigkeit erst initiieren. Freilich ist mit diesem Ergebnis, daß Leibniz in keinem philosophiehistorisch ergiebigen Sinn Eklektiker genannt werden kann, die Frage nicht vom Tisch, ob Eklektizismus eine philosophische Option war und wie Leibniz dazu stand. Denn rationalen Rekonstruktionen läßt sich, wie Hans Poser schrieb, durch historische Arbeit nur „begegnen", nicht eigentlich widersprechen. ${ }^{65}$ 


\section{Neuzeitliches Denken}

Festschrift für Hans Poser zum 65. Geburtstag

Herausgegeben von

Günter Abel Hans-Jürgen Engfer

Christoph Hubig

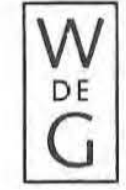

Walter de Gruyter · Berlin · New York onก? 